\title{
MONOTONICITY PROPERTIES OF LORENTZ SPACES
}

\author{
HENRYK HUDZIK AND ANNA KAMIŃSKA
}

(Communicated by Palle E. T. Jorgensen)

\begin{abstract}
Criteria for uniform monotonicity, local uniform monotonicity and strict monotonicity of Lorentz spaces are given.
\end{abstract}

Let $L^{0}$ denote the space of all (equivalence classes of ) Lebesgue measurable real-valued functions defined on the interval $[0, \gamma), \gamma \leq \infty$. In what follows, if $f, g \in L^{0}$, then $f \leq g$ means $f(t) \leq g(t)$ almost everywhere (a.e.) with respect to the Lebesgue measure $m$ on the real line. If $f \in L^{0}$ we denote by $d_{f}$ the distribution function of $|f|$, that is,

$$
d_{f}(t)=m\{s:|f(s)|>t\},
$$

and we denote by $f^{*}$ the decreasing rearrangement of $|f|$, that is,

$$
f^{*}(t)=\inf \left\{s>0: d_{f}(s) \leq t\right\} \text {. }
$$

Let $w:[0, \gamma) \rightarrow \mathbb{R}_{+}, \gamma \leq \infty$, be a nonincreasing, strictly positive, locally integrable function with respect to the Lebesgue measure $m$, called a weight function. Then the Lorentz space $\Lambda_{w}$ is defined as follows (see $[5,8,11,13]$ )

$$
\Lambda_{w}=\left\{f \in L^{0}:\|f\|=\int_{0}^{\gamma} f^{*}(t) w(t) d t=\int_{0}^{\gamma} f^{*} w<\infty\right\} .
$$

By $S(t)$ denote the function $S(t)=\int_{0}^{t} w(s) d s$. The weight $w(t)$ is called regular $[5,8,10]$ if $\inf _{t \in(0, \gamma)} \frac{S(2 t)}{S(t)}>1$.

Let $\left(E,\|\|_{E}\right)$ be a Banach function lattice over the measure space $(T, \Sigma, \mu)$ $[2,13,14] . E$ is said to be strictly monotone if for every $x, y \in E^{+}$(the positive part of $E), x \geq y,\|x\|_{E}=1$ and $y \neq 0$ imply that $\|x-y\|_{E}<1$. Following Birkhoff [2] we say that $E$ is uniformly monotone if for any $\epsilon \in(0,1)$ there exists $\delta(\epsilon) \in(0,1)$ such that $x, y \in E^{+}, y \leq x,\|x\|_{E}=1$ and $\|y\| \geq \epsilon$ imply $\|x-y\|_{E} \leq 1-\delta(\epsilon)$. The space $E$ is called locally uniformly monotone if for any $\epsilon \in(0,1)$ and $x \in E^{+}$with $\|x\|=1$, there exists $\delta(\epsilon, x) \in(0,1)$ such that $\|x-y\|_{E} \leq 1-\delta(\epsilon, x)$ whenever $y \in E^{+}, y \leq x$ and $\|y\|_{E} \geq \epsilon$.

Obviously uniform monotonicity implies local uniform monotonicity and local uniform monotonicity implies strict monotonicity.

Received by the editors January 14, 1994.

1991 Mathematics Subject Classification. Primary 46E30.

This research was done while the first named author was visiting Memphis State University. 
Monotonicity properties play in Banach lattices the analogous role as rotundity in Banach spaces. They are applicable among others to the dominated best approximation problems (see e.g. [4,9,12]) and to ergodic theory (see [1]). Those properties, with some of their applications, for Orlicz spaces for both the Orlicz and the Luxemburg norm were considered by Bru and Heinich in $[3,4]$, and by Medzhitov and Sukochev in [15]. In Musielak-Orlicz spaces, they were studied by Kurc in [12] and Hudzik and Kurc in [9]. Recently, the monotonicity properties of Calderon-Lozanovskii spaces were investigated in [6].

It is known that any uniformly convex Banach lattice $E$ is uniformly monotone. The Lorentz spaces $\Lambda_{p, w}(1<p<\infty)$, defined as the set of those $f \in L^{0}$ for which $\|f\|_{\Lambda_{p, w}}^{p}=\int_{0}^{\gamma}\left(f^{*}\right)^{p} w<\infty$, are uniformly convex and so uniformly monotone as well, if the weight function $w$ is regular (see [8]). This result was extended later to Orlicz-Lorentz spaces in [10]. For $p=1, \Lambda_{1, w} \equiv \Lambda_{w}$ is not strictly convex. However, the space $L^{1}$ ( a particular case of $\Lambda_{w}$ when $w(t) \equiv 1)$ is uniformly monotone, being flat with no extreme points. In this paper we show that $\Lambda_{w}$ is uniformly monotone whenever $w$ is a regular function. The necessity of regularity of $w$ is also proven. Strict and local uniform monotonicities of $\Lambda_{w}$ are also studied.

The theorems stated below are the main results of the paper.

Theorem 1. The Lorentz space $\Lambda_{w}$ is uniformly monotone if and only if the weight function $w$ is regular.

Theorem 2. $\Lambda_{w}$. is locally uniformly monotone whenever $\gamma<\infty$. If $\gamma=\infty$ and $\int_{0}^{\gamma} w<\infty$, then $\Lambda_{w}$ is not strictly monotone.

The proof of Theorem 1 will be preceded by two lemmas.

Lemma 1. Let the weight $w$ be a regular function. Then for every $p \in\{0\} \cup \mathbb{N}$ there exists a constant $k(p)>0$ such that

$$
S\left(2^{p} a+t\right)-S\left(2^{p} a\right) \geq k(p) S(t)
$$

for every $t \geq a$. If $t<a$ and $2^{p} t \geq a$ for some $p \in\{0\} \cup \mathbb{N}$, then

$$
S(t+a)-S(a) \geq k(p) S(t) .
$$

Proof. If $t \geq a$, then by monotonicity of $w$,

$$
S\left(2^{p} a+t\right)-S\left(2^{p} a\right) \geq S\left(\left(2^{p}+1\right) t\right)-S\left(2^{p} t\right) .
$$

Moreover, by regularity of the weight,

$$
S\left(2^{p+1} t\right)-S\left(2^{p} t\right) \geq(k-1) S\left(2^{p} t\right) \geq(k-1)^{p+1} S(t)
$$

and

$$
2^{p}\left(S\left(\left(2^{p}+1\right) t\right)-S\left(2^{p} t\right)\right) \geq S\left(2^{p+1} t\right)-S\left(2^{p} t\right) \geq(k-1)^{p+1} S(t) .
$$

This implies that $S\left(2^{p} a+t\right)-S\left(2^{p} a\right) \geq k(p) S(t)$ with $k(p)=2\left(\frac{k-1}{2}\right)^{p+1}$.

If $t<a$ and $2^{p} t \geq a$, then $S(a+t)-S(a) \geq S\left(\left(2^{p}+1\right) t\right)-S\left(2^{p} t\right) \geq k(p) S(t)$.

Lemma 2. Let the weight $w$ be a regular function. Then for any $\epsilon>0$ there exists $\eta(\epsilon)>0$ such that for any simple function with compact support and any measurable set $A$, if $\|f\| \leq 1$ and $\left\|f \chi_{A}\right\| \geq \epsilon$, then

$$
\left\|f-f \chi_{A}\right\| \leq\|f\|-\eta(\epsilon) \text {. }
$$


Proof. Let $f$ be a simple function and $A$ a measurable set such that $\|f\| \leq 1$ and $\left\|f \chi_{A}\right\| \geq \epsilon$. In the first part of the proof assume that $f$ is nonincreasing, that is,

$$
f=\sum_{i=1}^{n} \alpha_{i} \chi_{\left[a_{i-1}, a_{i}\right]}
$$

where $\alpha_{1}>\alpha_{2}>\ldots>\alpha_{n}>0=\alpha_{0}$ and $0=a_{0}<a_{1}<\ldots<a_{n}$. Thus

$$
\|f\|=\sum_{i=1}^{n}\left(\alpha_{i}-\alpha_{i-1}\right) S\left(a_{i}\right) \leq 1
$$

Let $A_{i}=\left[a_{i-1}, a_{i}\right] \cap A$ for $i=1,2, \ldots, n$ and $A_{0}=\varnothing$. Denote

$$
\begin{aligned}
c_{i} & =\sum_{j=1}^{i} m A_{j}, \quad i=0,1, \ldots, n, \\
d_{i} & =\sum_{j=0}^{i} m\left(\left[a_{j-1}, a_{j}\right] \backslash A_{j}\right), \quad i=1,2, \ldots, n, \quad d_{0}=0 .
\end{aligned}
$$

Notice that $d_{i}+c_{i}=a_{i}$. By the assumption that $\left\|f \chi_{A}\right\| \geq \epsilon$,

$$
\begin{aligned}
\int\left(f \chi_{A}\right)^{*} w & =\int \sum_{i=1}^{n} \alpha_{i} \chi_{\left[c_{i-1}, c_{i}\right]} w \\
& =\sum_{i=1}^{n}\left(\alpha_{i}-\alpha_{i-1}\right) S\left(c_{i}\right) \\
& \geq \epsilon .
\end{aligned}
$$

We have $\left(f-f \chi_{A}\right)^{*}=\sum_{i=1}^{n} \alpha_{i} \chi_{\left[d_{i-1}, d_{i}\right]}$. Setting $F=f-\left(f-f \chi_{A}\right)^{*}$,

$$
\int F w=\sum_{i=1}^{n}\left(\alpha_{i}-\alpha_{i-1}\right)\left(S\left(a_{i}\right)-S\left(d_{i}\right)\right) \text {. }
$$

Choose $p \in \mathbb{N}$ such that

$$
\frac{1}{k^{p}} \leq \frac{\epsilon}{4}
$$

where $k$ is the constant from the regularity condition of $w$. Define the following subsets of natural numbers. Let

$$
N_{1}=\left\{i=1, \ldots, n: 2^{p} d_{i}<c_{i}\right\} \text { and } N_{0}=\left\{i=1, \ldots, n: 2^{p} d_{i} \geq c_{i}\right\} .
$$

Then let

$$
\begin{aligned}
& N_{2}=\left\{i \in N_{0}: c_{i} \geq d_{i}\right\}, \\
& N_{3}=\left\{i \in N_{0}: 2^{p} c_{i}<d_{i}\right\}, \\
& N_{4}=\left\{i \in N_{0}: c_{i}<d_{i} \text { and } 2^{p} c_{i} \geq d_{i}\right\} .
\end{aligned}
$$

The sets $N_{i}(i=1, \ldots, 4)$ are disjoint, and its union is equal to the set $\{1,2, \ldots, n\}$. Since $\|f\| \leq 1, a_{i} \geq c_{i}$ and $w$ is regular,

$$
\begin{aligned}
1 & \geq \sum_{N_{1}}\left(\alpha_{i}-\alpha_{i-1}\right) S\left(a_{i}\right) \geq \sum_{N_{1}}\left(\alpha_{i}-\alpha_{i-1}\right) S\left(c_{i}\right) \\
& \geq \sum_{N_{1}}\left(\alpha_{i}-\alpha_{i-1}\right) S\left(2^{p} d_{i}\right) \geq k^{p} \sum_{N_{1}}\left(\alpha_{i}-\alpha_{i-1}\right) S\left(d_{i}\right),
\end{aligned}
$$


which implies that

$$
\sum_{N_{1}}\left(\alpha_{i}-\alpha_{i-1}\right) S\left(d_{i}\right) \leq \frac{1}{k^{p}}<\frac{\epsilon}{4} .
$$

Since $c_{i} \geq d_{i}$ for $i \in \mathbb{N}_{2}$, and $a_{i}=c_{i}+d_{i}$, applying Lemma 1 to $a=d_{i}$ and $x=c_{i}$, we get

$$
\sum_{N_{2}}\left(\alpha_{i}-\alpha_{i-1}\right)\left(S\left(a_{i}\right)-S\left(d_{i}\right)\right) \geq k(p) \sum_{N_{2}}\left(\alpha_{i}-\alpha_{i-1}\right) S\left(c_{i}\right)
$$

For $i \in N_{3}, c_{i}<\frac{1}{2^{p}} d_{i}$ and, by the regularity of $w, S\left(\frac{1}{2^{p}} d_{i}\right) \leq \frac{1}{k^{p}} S\left(d_{i}\right)$. Thus

$$
\begin{aligned}
\sum_{N_{3}}\left(\alpha_{i}-\alpha_{i-1}\right) S\left(c_{i}\right) & \leq \sum_{N_{3}}\left(\alpha_{i}-\alpha_{i-1}\right) S\left(\frac{1}{2^{p}} d_{i}\right) \\
& \leq \frac{1}{k^{p}} \sum_{N_{3}}\left(\alpha_{i}-\alpha_{i-1}\right) S\left(d_{i}\right) \\
& \leq \frac{1}{k^{p}}<\frac{\epsilon}{4} .
\end{aligned}
$$

Finally, since $c_{i}<d_{i}$ and $2^{p} c_{i} \geq d_{i}$ for $i \in N_{4}$, by Lemma 1, we get

$$
\sum_{N_{4}}\left(\alpha_{i}-\alpha_{i-1}\right)\left(S\left(a_{i}\right)-S\left(d_{i}\right)\right) \geq k(p) S\left(c_{i}\right) .
$$

By virtue of (1), either $\sum_{N_{0}}\left(\alpha_{i}-\alpha_{i-1}\right) S\left(c_{i}\right) \geq \frac{\epsilon}{2}$ or $\sum_{N_{1}}\left(\alpha_{i}-\alpha_{i-1}\right) S\left(c_{i}\right) \geq \frac{\epsilon}{2}$. Assuming the second inequality, by (2) and (4), we get the estimation

$$
\begin{aligned}
\int F w & \geq \sum_{N_{1}}\left(\alpha_{i}-\alpha_{i-1}\right) S\left(a_{i}\right)-\sum_{N_{1}}\left(\alpha_{i}-\alpha_{i-1}\right) S\left(d_{i}\right) \\
& \geq \sum_{N_{1}}\left(\alpha_{i}-\alpha_{i-1}\right) S\left(c_{i}\right)-\sum_{N_{1}}\left(\alpha_{i}-\alpha_{i-1}\right) S\left(d_{i}\right) \\
& \geq \frac{\epsilon}{2}-\frac{\epsilon}{4}=\frac{\epsilon}{4} .
\end{aligned}
$$

Now assume that $\sum_{N_{0}}\left(\alpha_{i}-\alpha_{i-1}\right)\left(S\left(c_{i}\right) \geq \frac{\epsilon}{2}\right.$. Then, by (6),

$$
\frac{\epsilon}{2} \leq \sum_{N_{2} \cup N_{4}}\left(\alpha_{i}-\alpha_{i-1}\right) S\left(c_{i}\right)+\frac{\epsilon}{4},
$$

whence

$$
\sum_{N_{2} \cup N_{4}}\left(\alpha_{i}-\alpha_{i-1}\right) S\left(c_{i}\right) \geq \frac{\epsilon}{4} .
$$

This combined with (5) and (7) imply that

$$
\begin{aligned}
\int F w & \geq \sum_{N_{2} \cup N_{4}}\left(\alpha_{i}-\alpha_{i-1}\right)\left(S\left(a_{i}\right)-S\left(d_{i}\right)\right) \\
& \geq k(p) \sum_{N_{2} \cup N_{4}}\left(\alpha_{i}-\alpha_{i-1}\right) S\left(c_{i}\right) \\
& \geq k(p) \frac{\epsilon}{4} .
\end{aligned}
$$


Thus we showed that $\int F w \geq k(p) \frac{\epsilon}{4}$. Hence

$$
\left\|f-f \chi_{A}\right\|=\int\left(f-f \chi_{A}\right)^{*} w=\int f w-\int F w \leq\|f\|-k(p) \frac{\epsilon}{4} .
$$

The constant $\eta(\epsilon)=k(p) \frac{\epsilon}{4}$ depends only on $\epsilon$.

Now let $f$ be any simple function with compact support satisfying the assumptions of the lemma. There exists a measure-preserving transformation $\sigma$ : $\mathbb{R}_{+} \rightarrow \mathbb{R}_{+}$such that $f^{*}(t)=f(\sigma(t))$. Thus $\left(f-f \chi_{A}\right)^{*}=\left(f(\sigma)-f(\sigma) \chi_{\sigma^{-1}(A)}\right)^{*}=$ $\left(f^{*}-f^{*} \chi_{\sigma^{-1}(A)}\right)^{*}$ and $\left\|f \chi_{A}\right\|=\int\left(f^{*} \chi_{\sigma^{-1}(A)}\right)^{*} w=\left\|f^{*} \chi_{\sigma^{-1}(A)}\right\|$. Since $f^{*}$ is a nonincreasing simple function with $\left\|f^{*} \chi_{\sigma^{-1}(A)}\right\| \geq \epsilon$, by the first part of the proof, there exists $\eta(\epsilon)>0$ such that $\left\|f^{*}-f^{*} \chi_{\sigma^{-1}(A)}\right\| \leq\left\|f^{*}\right\|-\eta(\epsilon)=$ $\|f\|-\eta(\epsilon)$. Now, the equality $\left\|f-f \chi_{A}\right\|=\left\|f^{*}-f^{*} \chi_{\sigma^{-1}(A)}\right\|$ completes the proof of the lemma.

Proof of Theorem 1. Assume that $w$ is a regular function, and let $f, g$ be simple functions with compact supports such that $\|f\| \leq 1,0 \leq g \leq f$ and $\|g\| \geq \epsilon$. Define

$$
A=\left\{t \in \mathbb{R}_{+}: g(t) \leq \frac{\epsilon}{2} f(t)\right\} .
$$

Then $\epsilon \leq\|g\| \leq \frac{\epsilon}{2}+\left\|g \chi_{A^{c}}\right\|\left(A^{c}\right.$ is a complement to $\left.A\right)$, whence $\left\|f \chi_{A^{c}}\right\| \geq$ $\left\|g \chi_{A^{c}}\right\| \geq \frac{\epsilon}{2}$. Moreover,

$$
f(t)-g(t) \leq f(t) \chi_{A}(t)+\left(f(t)-\frac{\epsilon}{2} f(t)\right) \chi_{A^{c}}(t)=f(t)-\frac{\epsilon}{2} f(t) \chi_{A^{c}}(t) .
$$

By Lemma 2, $\left\|f-f \chi_{A^{c}}\right\| \leq\|f\|-\eta\left(\frac{\epsilon}{2}\right)$. Thus

$$
\begin{aligned}
\|f-g\| & \leq\left\|f-\frac{\epsilon}{2} f \chi_{A^{c}}\right\| \leq\left(1-\frac{\epsilon}{2}\right)\|f\|+\frac{\epsilon}{2}\left\|f-f \chi_{A^{c}}\right\| \\
& \leq\left(1-\frac{\epsilon}{2}\right)+\frac{\epsilon}{2}\left(1-\eta\left(\frac{\epsilon}{2}\right)\right)=1-\frac{\epsilon}{2} \eta\left(\frac{\epsilon}{2}\right) .
\end{aligned}
$$

Finally, since the set of simple functions with compact supports is dense in $\Lambda_{w}$, the norm is uniformly monotone.

Now we shall show that the weight $w$ must be regular if $\Lambda_{w}$ is uniformly monotone. For any $u>0$, choose $a>0$ such that $\left\|a \chi_{[0,2 u]}\right\|=a S(2 u)=1$. Setting $f=a \chi_{[0,2 u]}$ and $g=a \chi_{[u, 2 u]}$ we have $0 \leq g \leq f,\|f\|=1$ and $\|g\|=a S(u) \geq \frac{1}{2} a S(2 u)=\frac{1}{2}$. By uniform monotonicity, there exists $\eta>0$ such that $\|f-g\| \leq 1-\eta$. However, $\|f-g\|=a S(u)$ and so $a S(u) \leq$ $1-\eta=(1-\eta) a S(2 u)$. Hence $S(u) \leq(1-\eta) S(2 u)$, which implies that $S(2 u) \geq k S(u)$, with $k=\frac{1}{1-\eta}>1$. Thus $w$ is regular and the proof is finished.

Proof of Theorem 2. If $\int_{0}^{\infty} w<\infty$, then taking $f \equiv 1$ and $g=\chi_{[1, \infty)}$, both functions belong to $\Lambda_{w}, f>g$ but $f^{*}=g^{*} \equiv 1$, which imply that $\Lambda_{w}$ is not strictly monotone.

Now assume that $\gamma<\infty$. We will show that $\Lambda_{w}$ is locally uniformly monotone. Here it will be convenient to use an equivalent sequence definition of local uniform monotonicity. Assuming $0 \leq f_{n} \leq f \in \Lambda_{w},\|f\|=1$ and $\left\|f_{n}\right\| \rightarrow\|f\|=1$ we need to show that $\left\|f-f_{n}\right\| \rightarrow 0$. At first we will prove that $\left\|f^{*}-f_{n}^{*}\right\| \rightarrow 0$. Indeed $\int_{0}^{\gamma}\left(f^{*}-f_{n}^{*}\right) w=\|f\|-\left\|f_{n}\right\| \rightarrow 0$, which in turn implies that there exists a subsequence $\left(n_{k}\right)$ of natural numbers such that $f_{n_{k}}^{*} \rightarrow f^{*}$ 
a.e. Since $d_{f_{n_{k}}^{*}}(t) \leq d_{f^{*}}(t)<\infty$ for every $t>0$, we get $\left(f^{*}-f_{n_{k}}^{*}\right)^{*}(t) \rightarrow 0$ for any $t>0$, by Proposition $12^{0}$ in [11, Chapter II, Paragraph 2]. Moreover, $\left(f^{*}-f_{n_{k}}^{*}\right)^{*} w \leq 2 f^{*} w$ and $\int_{0}^{\gamma} f^{*} w<\infty$. Therefore, by the Lebesgue dominated theorem, $\left\|f^{*}-f_{n_{k}}^{*}\right\|=\int_{0}^{\gamma}\left(f^{*}-f_{n_{k}}^{*}\right)^{*} w \rightarrow 0$.

Now, by a renorming result in [7], there exists a symmetric equivalent norm \|\|$_{0}$ in $\Lambda_{w}$ that is locally uniformly rotund (for definition of local uniform rotundity, see e.g. [13]). Set $\alpha=\frac{1}{\|f\|_{0}}$. By the first part of the proof, since $0 \leq \frac{f_{n}+f}{2} \leq f$ and $\left\|\frac{f_{n}+f}{2}\right\| \rightarrow\|f\|=1$, we get $\left\|\left(\frac{f_{n}+f}{2}\right)^{*}-f^{*}\right\| \rightarrow 0$. Therefore $\left\|\alpha\left(\frac{f_{n}+f}{2}\right)^{*}-\alpha f^{*}\right\|_{0} \rightarrow 0$. Consequently $\left\|\alpha f_{n}+\alpha f\right\|_{0}=\left\|\alpha\left(f_{n}+f\right)^{*}\right\|_{0} \rightarrow$ $2 \alpha\left\|f^{*}\right\|_{0}=2$. Since $\left\|\alpha f_{n}\right\|_{0} \leq\|\alpha f\|_{0}=1$ and \|\|$_{0}$ is locally uniformly rotund, $\left\|\alpha f_{n}-\alpha f\right\|_{0} \rightarrow 0$ and equivalently $\left\|f_{n}-f\right\| \rightarrow 0$, which completes the proof of theorem.

Remarks. The growth condition defining regularity of $w$ can be expressed equivalently as follows. For every $l>1$ there exists $k(l)>1$ such that $S(l t) \geq k(l) S(t)$ for all $t \geq 0$. Indeed, for $l \in(1,2), \int_{t}^{2 t} w \leq \frac{1}{l-1} \int_{t}^{l t} w$ by monotonocity of $w$. Assuming that $S(2 t) \geq k S(t)$,

$$
\begin{aligned}
S(l t) & =S(t)+\int_{t}^{l t} w \geq S(t)+(l-1) \int_{t}^{2 t} w \\
& =S(t)+(l-1)(S(2 t)-S(t)) \geq S(t)+(l-1)(k S(t)-S(t)) \\
& =k(l) S(t),
\end{aligned}
$$

with $k(l)=(1+(l-1)(k-1))>1$. The opposite implication is obvious.

One can ask whether it is possible to express the regularity of $w$ in terms of the function $w$ itself. We can easily show that if $w$ is regular, then there exists a constant $l \in(0,1)$ such that $w(2 t) \geq l w(t)$ for all $t \in \mathbb{R}_{+}$. Moreover, if $w$ satisfies the last inequality with $l \in\left(\frac{1}{2}, 1\right)$, then $w$ is regular. However, there are examples of functions that are not regular but satisfy the inequality $w(2 t) \geq \frac{1}{2} w(t)$ for all $t \in \mathbb{R}_{+}$. For instance, take $w(t)=1$ for $t \in[0,1]$ and $w(t)=\frac{1}{t}$ when $t>1$.

\section{REFERENCES}

1. M. A. Akcoglu and L. Sucheston, On uniform monotonicity of norms and ergodic theorems in function spaces, Rend. Circ. Mat. Palermo (2) Suppl. 8 (1985), 325-335.

2. G. Birkhoff, Lattice theory, Amer. Math. Soc., Providence, RI, 1967.

3. B. Bru and H. Heinich, Monotonies des espaces d'Orlicz, C. R. Acad. Sci. Paris Sér. I Math. 301 (1985), 893-894.

4. __ Applications de dualite dans les espaces de Köthe, Studia Math. 93 (1989), 41-69.

5. N. L. Carothers, Rearrangement invariant subspaces of Lorentz function spaces, Israel J. Math. 40 (1981), 217-228.

6. J. Cerda, H. Hudzik, and M. Mastylo, On the geometry of some Calderón-Lozanovskii interpolation spaces, Indag. Math. (to appear).

7. W. J. Davis, N. Ghossoub, and J. Lindenstrauss, A lattice renorming theorem and applications to vector-valued processes, Trans. Amer. Math. Soc. 263 (1981), 531-540.

8. I. Halperin, Uniform convexity in function spaces, Duke Math. J. 21 (1954), 195-204.

9. H. Hudzik and W. Kurc, Monotonicity properties of Musielak-Orlicz spaces equipped with the Orlicz norm (to appear). 
10. A. Kamińska, Uniform convexity of generalized Lorentz spaces, Arch. Math. 56 (1991), 181-188.

11. S. G. Krein, Yu. J. Petunin, and E. M. Semenov, Interpolation of linear operators, Transl. Math. Monographs, vol. 54, Amer. Math. Soc., Providence, RI, 1982.

12. W. Kurc, Strictly and uniformly monotone Musielak-Olricz spaces and applications to best approximation, J. Approx. Theory 69 (1992), 173-187.

13. J. Lindenstrauss and L. Tzafriri, Classical Banach spaces II, Springer-Verlag, Berlin, Heidelberg, and New York, 1979.

14. W. A. J. Luxemburg and A. Zaanen, Riesz spaces I, North-Holland, Amsterdam and London, 1971.

15. A. Medzhitov and Ph. Sukochev, The property $(H)$ in Orlicz spaces, Bull. Polish Acad. Sci. Math. 40 (1992), 5-11.

Department of Mathematics, A. Mickiewicz University, Poznań, Poland

E-mail address: Hudzik@plpuam11.bitnet

Department of Mathematical Sciences, The University of Memphis, Memphis, TenNESSEE 38152

E-mail address: Kaminska@hermes.msci.memst .edu 